\title{
Clinical effects of the bridge-type ROI-C interbody fusion cage system in the treatment of cervical spondylosis with osteoporosis
}

This article was published in the following Dove Press journal:

Clinical Interventions in Aging

\author{
Yuluo Rong* \\ Yongjun Luo* \\ Wei Liu \\ Fangyi Gong \\ Pengyu Tang \\ Weihua Cai
}

Department of Orthopaedics, The First Affiliated Hospital of Nanjing Medical University, Nanjing 210029 , Jiangsu, China

*These authors contributed equally to this work
Correspondence: Weihua Cai Department of Orthopaedics, The First Affiliated Hospital of Nanjing Medical University, Nanjing 210029, Jiangsu, China Tel +86 I395166 I0I8

Email caiwhspine@sina.com
Objective: To investigate the early and mid-term efficacy and safety of the bridge-type ROI-C interbody fusion cage system in the treatment of cervical spondylosis with osteoporosis during anterior cervical discectomy and fusion (ACDF).

Patients and methods: The clinical data from 24 cervical spondylosis patients with osteoporosis treated with ACDF were retrospectively analyzed. All patients were treated with ROI-C cage. Double-energy X-ray absorptiometry (DEXA) was used to measure the bone mineral density (BMD). The cervical sagittal radiographic parameters were measured and compared using X-ray including C2-C7 Cobb angle, segmental angle (SA), cervical vertebral bow depth, and height of operation segment (HOS). Postoperative dysphagia was recorded according to the Bazaz score. The Japanese Orthopedic Association (JOA) scores and Neck Disability Index (NDI) scores were used to evaluate the clinical outcomes at different time points. Odom and Vaccaro criteria were used to assess the surgical effects and to evaluate the fusion of the bone graft.

Results: The mean duration of the postoperative follow-up was 27.4 \pm 5.7 months (ranging from 21 to 36 months). The JOA scores and NDI scores at 3 months post operation and at the time of final follow-up were significantly different from those before surgery $(P<0.05)$. Two patients had mild dysphagia after surgery, but it disappeared after symptomatic treatment for 3-5 days. Sagittal radiographic outcomes were significantly improved at 3 months post operation $(P<0.05)$. At the time of final follow-up, the radiographic parameters were well maintained and were not significantly different compared with 3 months post operation $(P>0.05)$.

Conclusion: The ROI-C cage system is safe and effective for use in patients undergoing anterior cervical spondylosis surgery for osteoporosis treatment. It results in a positive effect on bone graft fusion, is able to effectively improve cervical curvature, restores intervertebral height, and reduces the incidence of postoperative dysphagia. The clinical effects were positive at the early and middle postoperative stages.

Keywords: cervical spondylosis, ROI-C, osteoporosis, imaging parameters, dysphagia

\section{Introduction}

Due to an aging population, the number of elderly patients with cervical spondylosis, a condition that is often accompanied by osteoporosis, is increasing. Many of these patients undergo degenerative changes in the cervical spine, which may be in need of cervical fixation and fusion surgery. Studies have found that $2 \%$ of patients undergoing surgery for cervical spondylosis are associated with osteoporosis, of whom the rate of anterior cervical spine surgery is $63.35 \%$ and the postoperative revision rate is $3.57 \%$, which is significantly higher than those without osteoporosis. ${ }^{1}$ With the development 
and improvement in cervical cage technologies, anterior cervical discectomy and fusion (ACDF) is currently widely used and is considered the gold standard for the treatment of cervical spondylosis. ${ }^{2,3}$ Among them, the bridge-type ROI-C interbody fusion device can reduce operation time, blood loss, postoperative dysphagia, adjacent segment degeneration, and other complications. Thanks to its precise clinical curative effect, it has been widely applied in clinical use. ${ }^{4,5} \mathrm{Up}$ to the present, no studies have reported on the use of bridge-type ROI-C interbody fusion cages for anterior cervical fusion with osteoporosis. This study retrospectively analyzed 24 patients with cervical spondylosis treated with ACDF using the bridge-type ROI-C interbody fusion cage system in our hospital from August 2013 to January 2015. The clinical results are reported in the following sections.

\section{Patients and methods}

\section{Research objectives}

In total, 24 patients with cervical spondylosis, accompanied with osteoporosis, were treated with the ROI-C interbody fusion cage in our hospital from August 2013 to January 2015. All patients gave written informed consent for participation in this study. This study was approved by the research ethics board of the First Affiliated Hospital of Nanjing Medical University. All patients were diagnosed with osteoporosis by double-energy X-ray absorptiometry (DEXA). Among them, there were nine cases of cervical spondylotic myelopathy, 13 cases of cervical spondylopathy, and two cases of mixed cervical spondylosis. The patients consisted of nine males and 15 females, with an average age of 58.2 years (ranging from 53 to 76 years). The average duration of postoperative follow-up was 27.4 months (ranging from 21 to 36 months). All cervical lesions were at the $\mathrm{C}_{3-7}$ level and included 19 cases of lesions in a single segment, four in double segments, and one in three segments. There were five cases of lesions in the $\mathrm{C}_{3-4}$ segment, ten in the $\mathrm{C}_{4-5}$ segment, 12 in the $\mathrm{C}_{5-6}$ segment, and three in the $\mathrm{C}_{6-7}$ segment (Table 1). One patient underwent cervical fusion surgery in the adjacent segment, and this lesion was in the upper segment of the fused segment. All patients underwent cervical spine X-ray, computed tomography (CT), and magnetic resonance imaging $(\mathrm{MRI})$ before surgery.

\section{Inclusion criteria}

Inclusion criteria were as follows: the presence of MRI and other imaging examination evidence to suggest that the spinal cord or nerve roots were compressed; presentation with obvious corresponding clinical symptoms, such as neck and
Table I Descriptive data, bone density, and operation details of patients

\begin{tabular}{l|l}
\hline & ROI-C \\
\hline Mean age (years) & $58.2 \pm 6.8$ \\
Number of samples & 24 \\
Gender & 9 \\
$\quad$ Male & 15 \\
$\quad$ Female & $27.4 \pm 5.7$ \\
Duration of follow-up (months) & -2.6 \\
Bone density & \\
Operation level & 19 \\
$\quad$ Level I & 4 \\
Level 2 & 1 \\
Level 3 & \\
Operation time (months) & $57 \pm 19$ \\
Level I & $93 \pm 27$ \\
Level 2 & 128 \\
Level 3 & \\
\hline
\end{tabular}

Note: Data shown as number or mean \pm standard deviation.

shoulder pain, numbness, tightness in the chest, limb weakness, walking instability, foot cotton, and other symptoms; a bone density examination revealing osteoporosis $(\mathrm{T} \leq-2.0)$; symptoms that were not significantly relieved and still affected normal life after 6 months of regular conservative treatment; and complete medical records and imaging data. Patients with severe cervical instability, cervical fracture and dislocation, spinal stenosis, tumor, and severe osteoporosis were excluded from the study.

\section{Clinical evaluation indicators}

The clinical outcomes were assessed using the Japanese Orthopedic Association (JOA) scores ${ }^{6}$ and Cervical Dysfunction Index (NDI) scores ${ }^{7}$ before and after the surgery. Odom's method was used to determine the efficacy of the surgery and was ranked as follows: excellent, postoperative symptoms and signs disappeared; good, most of the symptoms and signs were relieved and normal functioning was returned; fair, symptoms and signs were partially improved, but they could not function properly; poor, symptoms and signs were basically the same as before surgery. According to the Bazaz score, ${ }^{8}$ the severity of postoperative dysphagia was graded as follows: none, no swallowing problems; mild, almost no dysphagia; moderate, occasional dysphagia; and severe, frequent dysphagia.

\section{Radiological assessment}

All patients underwent standard cervical radiography before surgery, 3 months after surgery, and at the time of last follow-up. The C2-C7 Cobb angle, ${ }^{9}$ segmental angle (SA), and height of operation segment (HOS) were measured, 

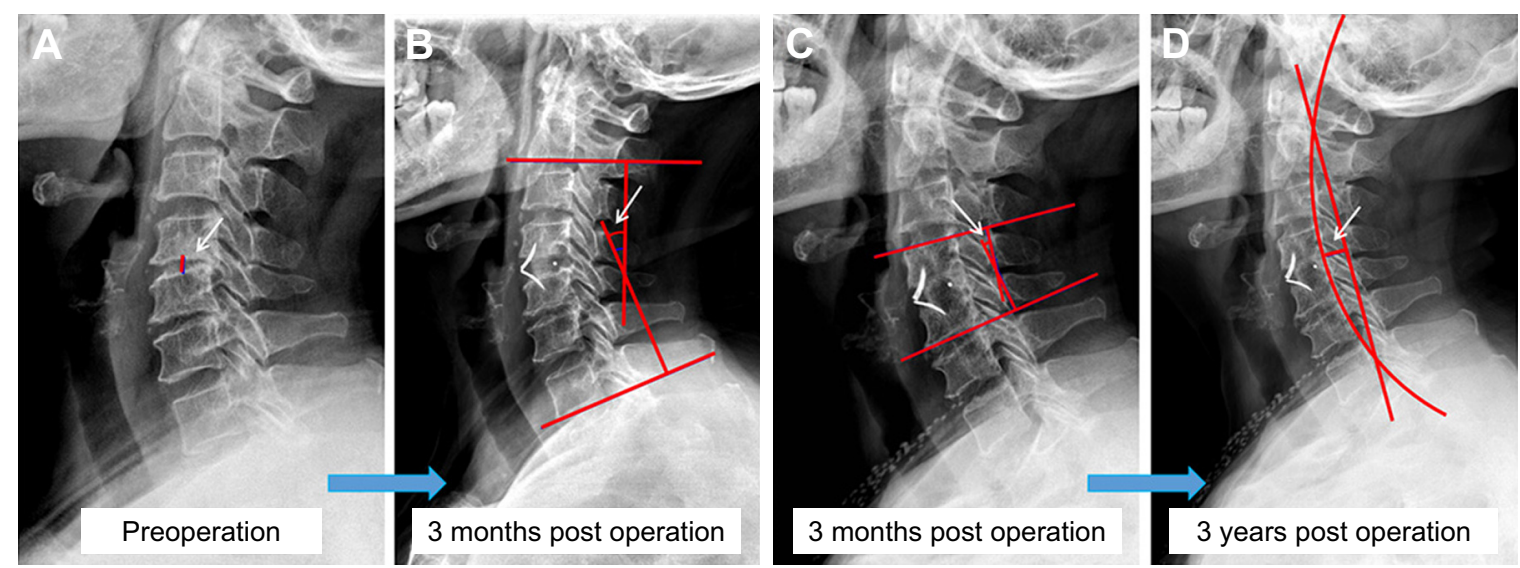

Figure I Methods of measuring cervical radiographic parameters $(\mathrm{T}=-2.7)$.

Notes: (A) Height of the operation segment, measured as the distance between the midpoint of the upper end plate and the midpoint of the lower end plate. (B) The cervical $\mathrm{C} 2-\mathrm{C7}$ Cobb angle is the angle between the perpendicular line of the inferior end plate of the $\mathrm{C} 2$ and $\mathrm{C7}$ vertebrae. A positive value denotes lordosis, and a negative value denotes kyphosis. (C) The SA is the angle between the perpendicular line of the superior end plate of the cranial vertebrae and the inferior end plate of the caudal vertebrae. (D) The cervical vertebral bow depth is measured as the line between the upper edge of the posterior odontoid process and the posterior and lower edge of the C7 vertebra, connecting the posterior margin of the vertebrae to the arc and the vertical distance from the arc top to the front line.

Abbreviation: SA, segmental angle.

and Borden's method was used for the measurement of the cervical vertebral bow depth (Figure 1). The Vaccaro standard was used to evaluate the status of interbody fusion. ${ }^{10}$ The fusion criteria were as follows: displacement angle of the cervical vertebral motility position adjacent to the vertebral body did not exceed $2^{\circ}$; height of the intervertebral space was recovered without collapse; bone trabecular and upper and lower vertebrae possessed bony connection; no translucent line was visible between the bone area and the upper and lower edges of the vertebral body.

\section{Materials}

The curved area above the ROI-C fusion cage (LDR, Troyes, France) follows the natural vertebral anatomy (Figure 2). Its leading edge is higher than the posterior edge, bringing the bone and the implant in close contact with each other. It can effectively restore and maintain the height of the intervertebral space and the cervical physiological curvature. It also provides a large cross-sectional area, increases contact area between the bone graft and the upper and lower vertebral bodies, promotes fusion, and reduces the risk of collapse. The outer bearing surface is large, and its serrated appearance and anatomical form increase the initial stability. Different models and specifications are available which are suitable for different anatomical features of the spine, so that it can be more perfectly matched with cervical anatomy. Polyetheretherketone (PEEK) composites permit the penetration of X-rays, allowing for the evaluation of bone growth and fusion. ${ }^{11,12}$

\section{Surgical methods}

With the patient in a supine position, the classic Robinson and Cloward anterior cervical approach and technique ${ }^{13}$ were used. After completion of decompression, a bridge ROI-C fusion cage of suitable size was installed. Postoperative fluid analgesia and regular anti-osteoporosis treatment were performed. Two days after surgery, the drainage tube was removed. When the patient was able to get out of bed, a cervical collar was worn for 3 months after the operation.
A

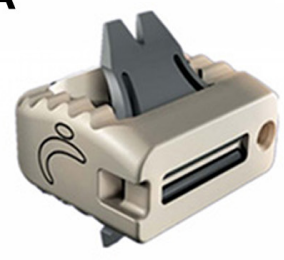

B

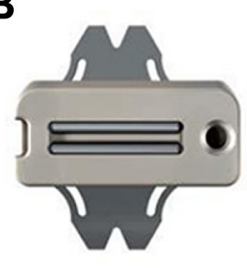

C

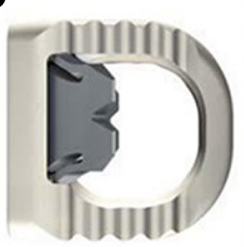

D

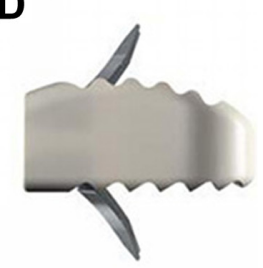

Figure 2 The bridge-type ROI-C interbody fusion cage system.

Notes: (A) Oblique view. (B) Anterior view. (C) Superior view. (D) Lateral view. 


\section{Statistical methods}

SPSS 20.0 software (IBM Corporation, Armonk, NY, USA) was used for statistical analysis of the data. Results of cervical radiological parameters are expressed as mean \pm standard deviation. A paired-sample $t$-test was used to compare changes in JOA scores, NDI scores, and radiological parameters before and after surgery. $P$-value of $\leq 0.05$ was regarded as statistically significant.

\section{Results}

\section{General conditions of patients}

Anesthesia for all patients was satisfactory, and operations were carried out successfully without any injury to the trachea, esophagus, dura mater, or spinal cord. No hoarseness of voice, dyspepsia, dyspnea, or other complications, such as deep vein thrombosis of the lower extremity, arose after the operation. All patients were discharged 3-5 days after operation and showed good incision healing. During the follow-up period, symptoms of spinal cord and nerve compression were significantly relieved compared with the preoperative state. At 3 months post operation, only two cases had poor bone graft fusion and the bone fusion rate was $91.7 \%$. At the final follow-up, all patients had achieved bone fusion, and no blade fractures, cage subsidence, or loosening was observed.

\section{Clinical and radiological results}

Twenty-four patients were diagnosed with osteoporosis by DEXA before the operation, with an average T-value of -2.6 . The average operation time was $57 \pm 19$ minutes on a single-segment case, $93 \pm 27$ minutes on a double-segment case, and 128 minutes on the single three-segment case. The patients were followed up for 21-36 months, for an average of $27 \pm 5.7$ months. The JOA score increased from the preoperative measurement $(9.2 \pm 1.8)$ to the time of final follow-up (13.8 \pm 1.6$)$ and NDI scores decreased from preoperative measurement (36.9 \pm 11.7 ) to the time of final follow-up (7.9 \pm 3.1$)$. The JOA and NDI scores at 3 months postoperatively and at the time of final follow-up were significantly different from those before surgery (Table $2 ; P<0.05$ ). The effect of surgical

Table 2 The JOA and NDI scores of patients at different time points

\begin{tabular}{l|l|l}
\hline & JOA score & NDI score \\
\hline Preoperation & $9.2 \pm 1.8^{\mathrm{a}}$ & $36.9 \pm 11.7^{\mathrm{a}}$ \\
3 months post operation & $13.1 \pm 1.9^{\mathrm{b}}$ & $14.2 \pm 10.6^{\mathrm{b}}$ \\
Final follow-up & $13.8 \pm 1.6^{\mathrm{b}}$ & $7.9 \pm 3.1^{\mathrm{b}}$ \\
\hline
\end{tabular}

Notes: b compared with a: $P<0.05$. Data shown as mean \pm standard deviation.

Abbreviations: JOA, Japanese Orthopedic Association; NDI, Cervical Dysfunction Index. treatment was determined by Odom's method, with $95.8 \%$ rated as excellent or good. Two patients suffered from mild dysphagia after surgery, which disappeared after 3-5 days of symptomatic treatment. The C2-C7 Cobb angle, SA, cervical vertebral bow depth, and HOS were significantly improved at 3 months post operation (Table $3 ; P<0.05$ ). At the final follow-up, the C2-C7 Cobb angle, SA, cervical vertebral bow depth, and HOS were well maintained, with no significant difference observed compared with the values obtained at 3 months post operation (Table $3 ; P>0.05$ ).

Typical cases were as follows: 1) a 66 year-old male patient was admitted to the hospital showing numbness of the limbs and walking instability for 2 years. The symptoms worsened over 3 months. DEXA results showed that the T-value of the lumbar spine bone density was -2.5 . Radiological data before and after surgery and upon follow-up are shown in Figures 3-5. 2) A 64 year-old male patient, who underwent anterior cervical surgery 3 years ago, suffered from degeneration of adjacent spinal segments after surgery. The diagnosis was cervical spondylotic myelopathy and adjacent segment degeneration of the cervical spine. The preoperative $\mathrm{T}$-value was -2.3 . The imaging data before and after surgery are shown in Figures 6 and 7.

\section{Discussion}

Osteoporosis, one of the most common human bone diseases, is characterized by reduced bone mass and deterioration of bone tissue and structure. ${ }^{14}$ The number of people with osteoporosis in USA is estimated at 10 million, and it is expected to increase to 20 million by $2020 .{ }^{15}$ In China, among people over 50 years old, approximately 69.44 million people suffer from osteoporosis. Among them, the prevalence rate of women reached $20.7 \%$, and that of men was $14.4 \% .{ }^{16}$ In 1994 , the WHO proposed diagnostic criteria for osteoporosis, which were based on data from white postmenopausal women. The criteria were as follows: a bone mineral density (BMD) at the affected site of 2.5 SD lower than that of the average - meaning a T-value of $\leq-2.5 \mathrm{SD}$. If accompanied by a brittle fracture, it is termed as severe osteoporosis. This standard is based on the T-value, which is calculated according to the following formula:

\section{BMD measured value -}

\section{$\mathrm{T}$-value $=\frac{\text { BMD normal youthpopulation reference value }}{\text { SD normal youth population reference value }}$}

Thus, the selection criteria of the normal reference population are key; the BMD of the normal reference population varies according to region, lifestyle, and dietary 
Table 3 Radiographic outcomes of patients at different time points: cervical curvature, SA, cervical vertebral bow depth, and HOS measurements

\begin{tabular}{|c|c|c|c|c|}
\hline & $\begin{array}{l}\text { Cervical curvature } \\
\text { (C2-C7 Cobb angle) }\left(^{\circ}\right)\end{array}$ & SA $\left({ }^{\circ}\right)$ & $\begin{array}{l}\text { Cervical vertebral } \\
\text { bow depth }(\mathrm{mm})\end{array}$ & $\begin{array}{l}\text { HOS } \\
(\mathrm{mm})\end{array}$ \\
\hline Preoperation & $12.9 \pm 7.8^{\mathrm{a}}$ & $2.3 \pm 5.4^{a}$ & $8.1 \pm 7.4^{a}$ & $4.9 \pm 1.8^{\mathrm{a}}$ \\
\hline 3 months post operation & $15.4 \pm 8.3^{b}$ & $4.8 \pm 5.9^{b}$ & $9.6 \pm 8.1^{b}$ & $7.2 \pm 1.3^{b}$ \\
\hline Final follow-up & $15.7 \pm 6.8^{b}$ & $4.9 \pm 6.5^{b}$ & $10.1 \pm 8.7^{b}$ & $7.1 \pm 0.9^{b}$ \\
\hline
\end{tabular}

Notes: b compared with a: $P<0.05$. Data shown as mean \pm standard deviation.

Abbreviations: HOS, height of operation segment; SA, segmental angle.

habits; thus, the diagnostic criteria do not fully apply to the Chinese population. In 1999, the Diagnostic Group of the Osteoporosis Committee of the Chinese Gerontology Society suggested that the diagnostic criterion for osteoporosis should be a loss of $25 \%$ of bone mass, or $2.0 \mathrm{SD}$, a standard that is more in-line with China's physical characteristics. Hee et $\mathrm{al}^{17}$ reported on the complications of 21 cases of osteoporotic multi-segmental cervical spondylosis treated with an anterior titanium mesh graft with titanium plate fixation. They achieved $95 \%$ bone graft fusion, but up to $33 \%$ of cases had complications, with the main cause of complications being a failure of the intervertebral cage and titanium mesh. Yan et a $\mathrm{l}^{18}$ conducted a single-center study on the use of titanium mesh and dynamic titanium plates in the treatment of subtotal vertebral resection in osteoporotic patients of varying degrees. They found no difference in neurological recovery and postoperative cervical curvature, but found striking differences in the degree of osteoporosis subsidence, with all patients showing satisfying bone fusion. Of course, regular anti-osteoporosis treatment based on the specific patient characteristics is necessary during the perioperative period, and the anti-osteoporosis treatment program should be adjusted according to the follow-up results. Wang et $\mathrm{a}^{19}$ studied the anterior fusion surgery of cervical spondylotic myelopathy with osteoporosis and assessed the effect of anti-osteoporosis treatment on the overall curative effect. Their results confirmed that anti-osteoporosis treatment could further improve cervical curvature and fusion segment intervertebral height and was also more effective in alleviating pain in the upper extremities. All patients in our study received regular anti-osteoporosis treatment, and all had symptoms that were remarkably alleviated. The JOA and NDI scores were significantly improved, and the efficacy was well maintained during the follow-up period of up to 3 years. Bone fusion was achieved in all surgical segments. The curvature of the cervical spine and intervertebral height were effectively maintained, and there was no failure of internal fixation. This indicates that interbody fusion cage treatment of cervical spondylosis combined with osteoporosis anterior fusion surgery can achieve a satisfying surgical outcome.

ACDF is a classical surgical treatment for cervical spondylosis. It can directly alleviate decompression,
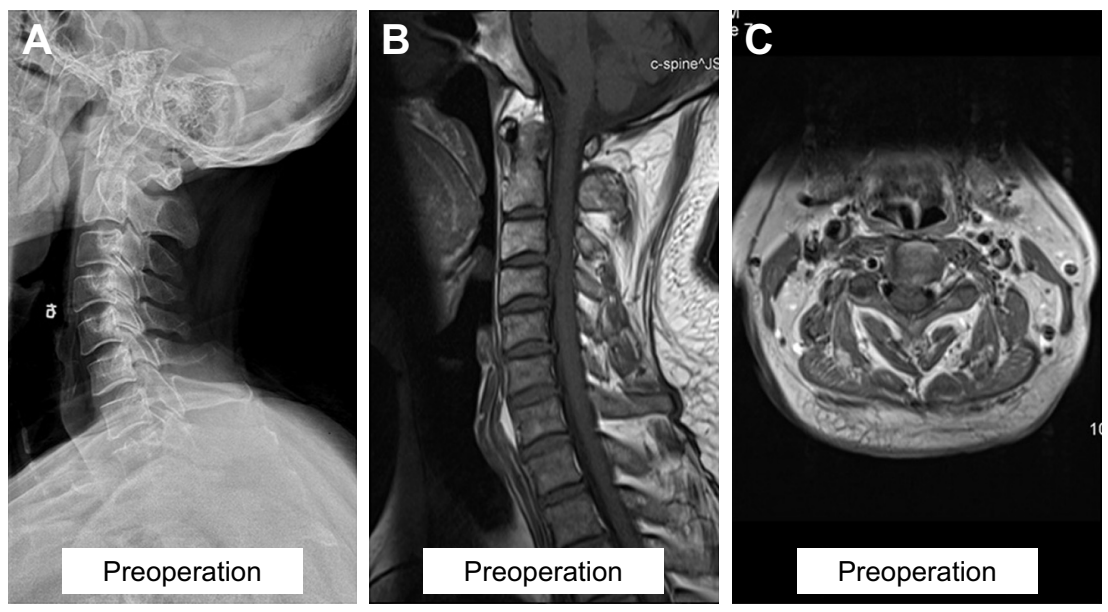

Figure 3 Preoperative imaging examination.

Notes: (A) X-ray image showing degeneration of the cervical vertebrae, narrowing of the intervertebral space, and decreased physiological bending of the cervical vertebrae. (B, C) MRI showing C3-C4 intervertebral disk herniation and significant compression of the dura mater.

Abbreviation: MRI, magnetic resonance imaging. 

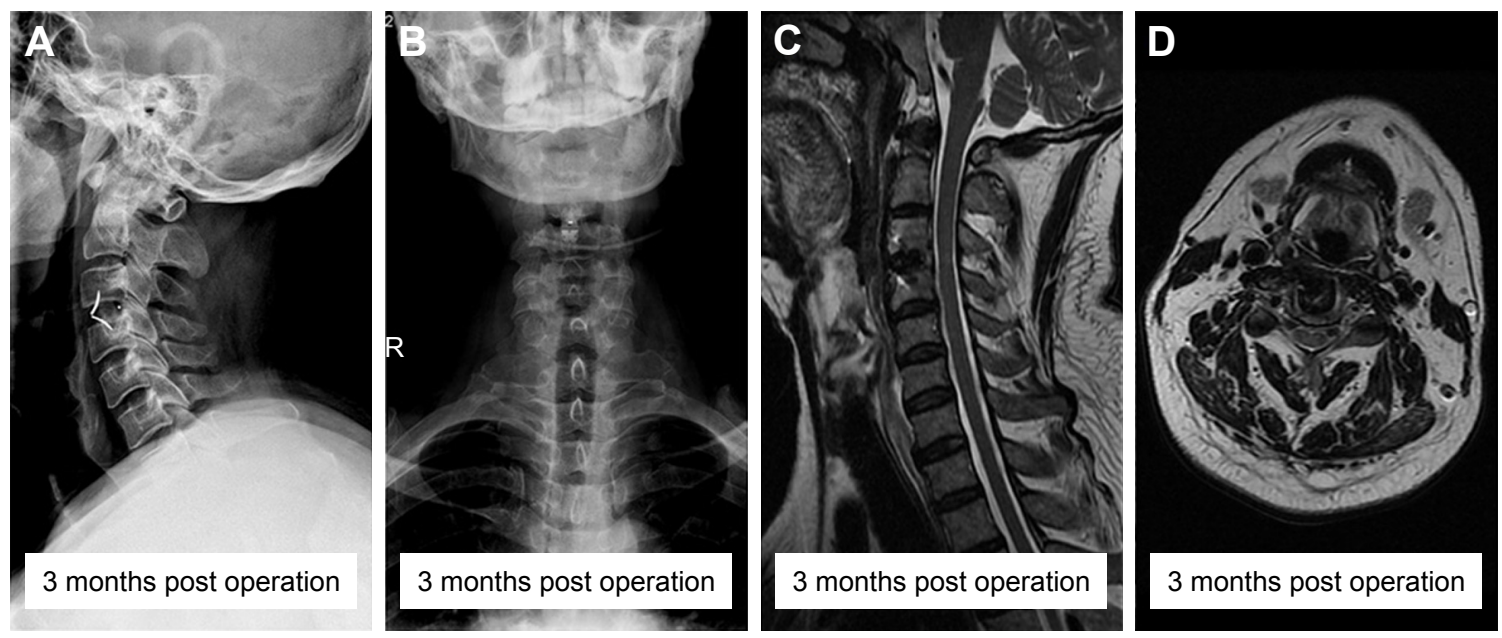

Figure 4 Imaging examination at 3 months post operation.

Notes: (A, B) X-ray image showing that the fusion device was in place in a satisfactory position. The lordosis of the cervical spine was well maintained. (C, D) MRI showing satisfactory decompression at the C3-C4 segment.

Abbreviation: MRI, magnetic resonance imaging.

effectively restoring cervical lordosis through distraction of the intervertebral space and rebuilding the stability of the diseased segment, resulting in clinically satisfactory results. ${ }^{2}$ In traditional cervical anterior surgery, fusion cage and titanium plate fixation systems are widely used. The use of titanium plates can increase the stability of the cervical spine, reduce the looseness of the fusion cage, and increase the interbody fusion rate. ${ }^{20}$ However, the use of titanium plates has also increased the range of anterior longitudinal ligament dissection, thus increasing the risk of esophageal or tracheal injury, nerve injury, and dysphagia. ${ }^{21}$ The bridge-type ROI-C interbody fusion cage, which is used in the clinic at present, consists of two teeth-like inserts and a
PEEK composite cage. An ACDF that uses the bridge ROI-C fusion device can simplify the surgical procedure, eliminating the need for screw drilling, tapping, and other steps in the anterior titanium plate surgery, thus saving surgical time and reducing surgical bleeding. ${ }^{5}$ In addition, there is no need for titanium plate fixation in front of the vertebral body, which has a smaller range of surgical exposure, leading to reduced pulling of the trachea, esophagus, and nerves, and a reduction in the incidence and severity of postoperative dysphagia. ${ }^{4}$ Regarding biomechanical stability, the bridge-type ROI-C fusion cage has the same efficacy as the fusion cage plus titanium plate. ${ }^{22}$ The bridge-type ROI-C fusion device is relatively simple to implant and brings certain advantages
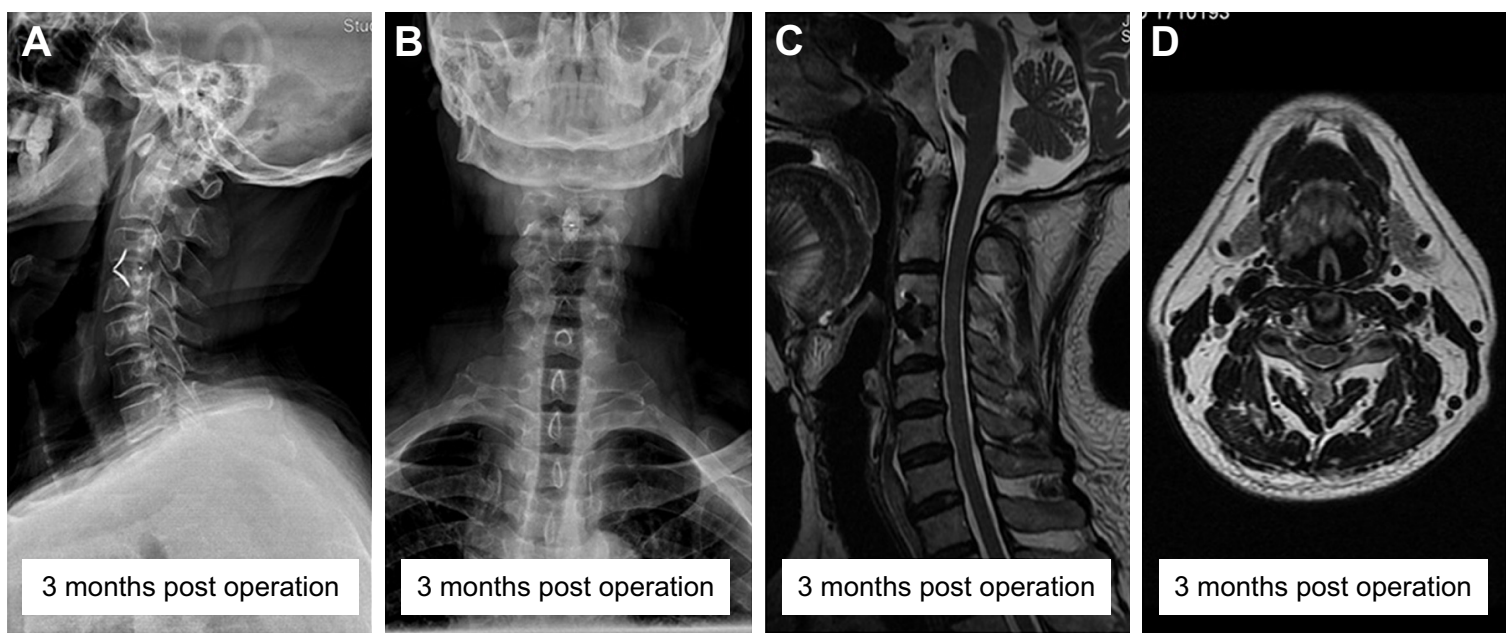

Figure 5 Imaging examination at 3 years post operation.

Notes: (A, B) X-ray image showing satisfactory position of the cage and good maintenance of cervical lordosis. (C, D) MRI showing satisfactory decompression at the C3-C4 segment and no increase in degeneration in the adjacent segment.

Abbreviation: MRI, magnetic resonance imaging. 

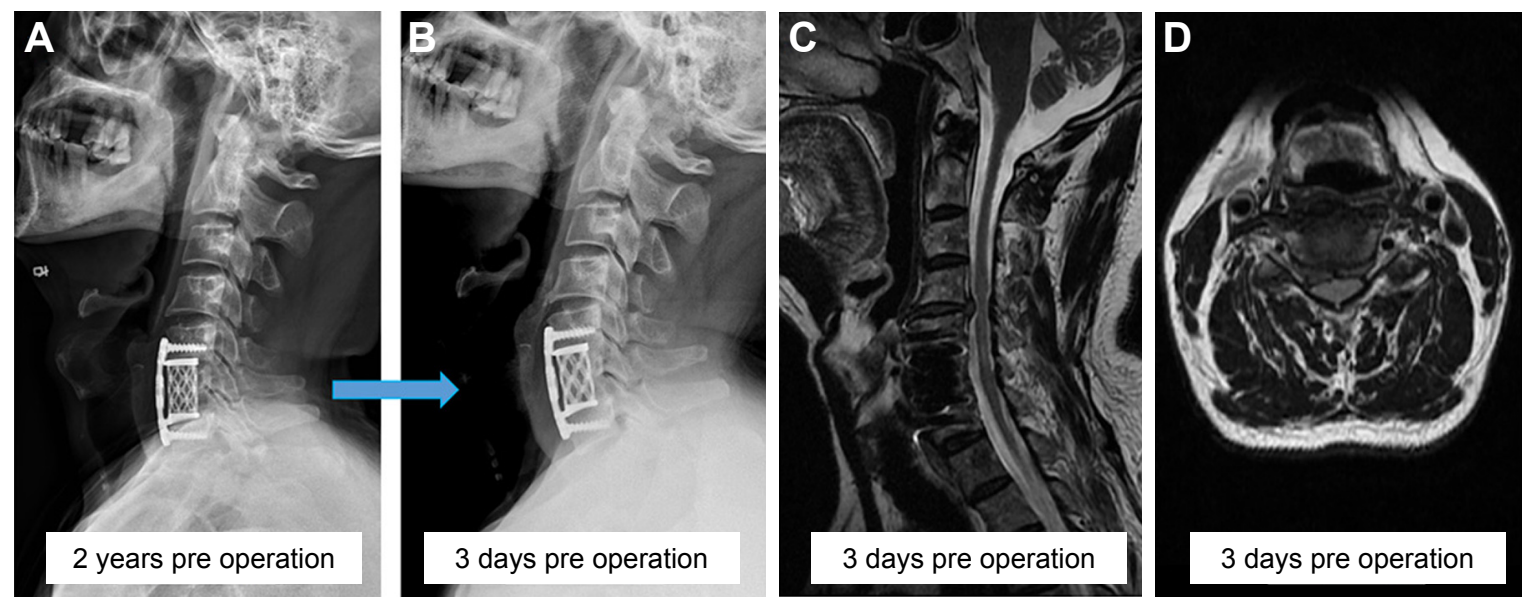

Figure 6 Preoperative imaging examination.

Notes: (A, B) X-ray image showing degeneration of the cervical vertebrae and narrowing and progressive aggravation of the intervertebral space in the adjacent segment. (C, D) MRI showing C4-C5 disk herniation in the segment adjacent to the operation with dura mater compression and spinal stenosis.

Abbreviation: MRI, magnetic resonance imaging.

to anterior cervical angioplasty, such as reduced removal of soft tissue and no need to replace screws and titanium plates. Based on the results of this study, we found that there was no sinking of the fusion cage, and the final surgical outcome was satisfactory in all cases. This demonstrates that the use of the bridge-type ROI-C interbody fusion cage system is safe and reliable, and even in cases of anterior fusion surgeries with osteoporosis in cervical spondylosis a satisfactory effect of bone graft fusion can be achieved, with the short- and mid-term curative effects still well maintained.

Osteoporosis is the most common degenerative human skeletal disease. Spinal surgeons will inevitably perform surgery on patients with osteoporosis, with surgery indications for elderly people at risk of osteoporosis similar to those without osteoporosis, including cervical nerve root, cervical spondylotic myelopathy, and cervical disk herniation. ${ }^{23}$ Due to the difficulty of the surgery and potential complications, such as nonunion fractures, adjacent horizontal fractures, and instrument failure, the treatment of patients with spinal osteoporosis is challenging. Currently, no studies have reported on the use of the bridge-type ROI-C interbody fusion cage system for cervical anterior fusion surgery with osteoporosis. Several previous studies ${ }^{5,21,24}$ have used the bridge-type ROI-C interbody fusion cage system in middleaged and elderly patients. Although osteoporosis alone was not proposed in these studies as a prognostic factor, bone fusion was achieved in almost all cases, and internal fixationrelated complications were extremely low. All these factors indirectly implicate the safety of the bridge-type ROI-C fusion cage system applied to anterior cervical fusion surgery
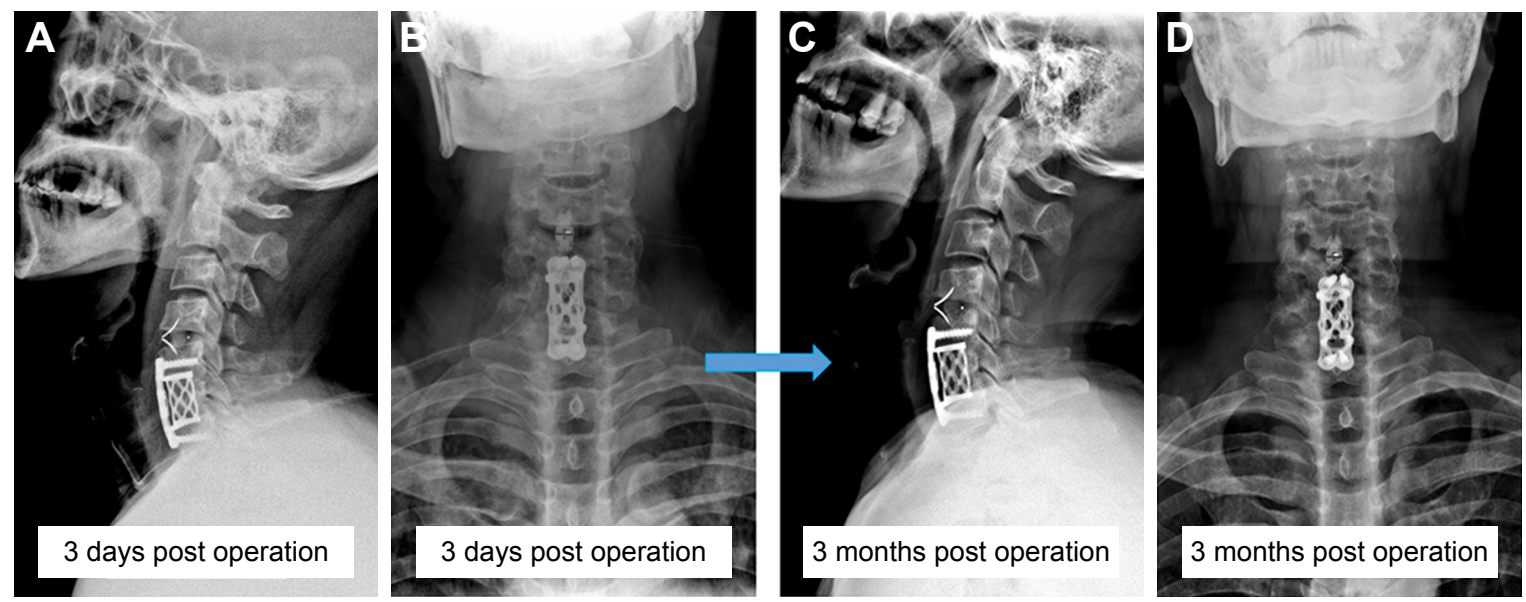

Figure 7 Postoperative imaging examination.

Notes: (A, B) X-ray imaging showing the satisfactory position of the fusion device at 3 days post operation. (C, D) X-ray imaging showing the satisfactory position of the fusion cage after 3 months. The cervical lordosis was well maintained. 
with osteoporosis. In addition, the ROI-C fusion device, which uses a new type of intervertebral bridge-type selfstabilizing technology, has a unique interface between the fusion device and the insert, a stress separation between the fusion device and the fixed plate, and the ability to lock the double inserts in one step. The large cross-sectional area increases the contact area between the bone graft and the upper and lower vertebral bodies, which promotes fusion and reduces the risk of collapse. The outer bearing surface is large, and the saw-tooth shape and anatomy can enhance initial stability. Even if loosening of the insert is caused by osteoporosis, it will not affect the stability of the cage; on the contrary, it can help reduce the risk of sinking of the cage and improve the safety of use. Osteoporotic fractures are associated with BMD - many prospective studies have shown that for every $1 \mathrm{SD}$ reduction in BMD, the risk of osteoporotic fractures is increased from 1.5 to 3.0 times. ${ }^{25}$ Therefore, it is not recommended to use this fusion device in elderly patients with severe osteoporosis, as loosening and sinking of the cage may occur due to poor bone quality. It also cannot be applied to patients with severe cervical instability and fracture dislocation, as the ligaments and bony structures are impaired, and the stability of the cervical segment cannot be guaranteed.

\section{Conclusion}

The use of the bridge-type ROI-C interbody fusion cage system in the treatment of cervical spondylosis with osteoporosis in anterior cervical surgery is safe and effective. It can improve the curvature of the cervical spine, the cervical vertebral bow depth, the curvature, and intervertebral height of the surgical segment, in addition to other benefits. However, the main drawback of this study is that the results were not compared with a control series of non-osteoporotic cervical spondylosis patients. An additional limitation of this study is that the nature of the analysis was retrospective and may be subject to bias. Further research is needed to avoid selection bias, and the long-term follow-up studies are needed to provide the best clinical data to determine the efficacy of the bridge-type ROI-C interbody fusion cage in the treatment of cervical spondylosis with osteoporosis during ACDF.

\section{Acknowledgment}

This study was sponsored by the Wu Jieping Foundation (grant no. 320-2745-16-117), and the "333 High Level Talents Project" in Jiangsu Province, China (grant no. BRA2016512).

\section{Disclosure}

The authors report no conflicts of interest in this work.

\section{References}

1. Guzman JZ, Feldman ZM, McAnany S, Hecht AC, Qureshi SA, Cho SK. Osteoporosis in Cervical Spine Surgery. Spine. 2016;41(8): 662-668.

2. Mummaneni PV, Haid RW. The future in the care of the cervical spine: interbody fusion and arthroplasty. Invited submission from the Joint Section Meeting on Disorders of the Spine and Peripheral Nerves, March 2004. J Neurosurg Spine. 2004;1(2):155-159.

3. Nabhan A, Ahlhelm F, Shariat K, et al. The ProDisc-C prosthesis: clinical and radiological experience 1 year after surgery. Spine. 2007; 32(18):1935-1941.

4. Bucci MN, Oh D, Cowan RS, et al. The ROI-C zero-profile anchored spacer for anterior cervical discectomy and fusion: biomechanical profile and clinical outcomes. Med Devices. 2017;10:61-69.

5. Lu Y, Bao W, Wang Z, et al. Comparison of the clinical effects of zero-profile anchored spacer (ROI-C) and conventional cage-plate construct for the treatment of noncontiguous bilevel of cervical degenerative disc disease (CDDD): A minimum 2-year follow-up. Medicine. 2018;97(5):e9808.

6. Yonenobu K, Abumi K, Nagata K, Taketomi E, Ueyama K. Interobserver and intraobserver reliability of the Japanese orthopaedic association scoring system for evaluation of cervical compression myelopathy. Spine. 2001;26(17):1890-1894.

7. Vernon H, Mior S. The Neck Disability Index: a study of reliability and validity. J Manipulative Physiol Ther. 1991;14(7):409-415.

8. Bazaz R, Lee MJ, Yoo JU. Incidence of dysphagia after anterior cervical spine surgery: a prospective study. Spine. 2002;27(22):2453-2458.

9. Mummaneni PV, Burkus JK, Haid RW, Traynelis VC, Zdeblick TA. Clinical and radiographic analysis of cervical disc arthroplasty compared with allograft fusion: a randomized controlled clinical trial. J Neurosurg Spine. 2007;6(3):198-209.

10. Vaccaro AR, Carrino JA, Venger BH, et al. Use of a bioabsorbable anterior cervical plate in the treatment of cervical degenerative and traumatic disc disruption. J Neurosurg. 2002;97(4 Suppl): 473-480.

11. Ferguson SJ, Visser JM, Polikeit A. The long-term mechanical integrity of non-reinforced PEEK-OPTIMA polymer for demanding spinal applications: experimental and finite-element analysis. Eur Spine J. 2006;15(2): 149-156.

12. Kulkarni AG, Hee HT, Wong HK, Cage S. Solis cage (PEEK) for anterior cervical fusion: preliminary radiological results with emphasis on fusion and subsidence. Spine J. 2007;7(2):205-209.

13. Whitecloud TS. Anterior Surgery for Cervical Spondylotic Myelopathy. Spine. 1988;13(7):861-863.

14. Cosman F, de Beur SJ, LeBoff MS, et al. Clinician's Guide to Prevention and Treatment of Osteoporosis. Osteoporos Int. 2014;25(10): 2359-2381.

15. Burge R, Dawson-Hughes B, Solomon DH, Wong JB, King A, Tosteson A. Incidence and economic burden of osteoporosis-related fractures in the United States, 2005-2025. J Bone Miner Res. 2007;22(3): 465-475.

16. Takahara K, Kamimura M, Moriya H, et al. Risk factors of adjacent vertebral collapse after percutaneous vertebroplasty for osteoporotic vertebral fracture in postmenopausal women. BMC Musculoskelet Disord. 2016;17:12.

17. Hee HT, Majd ME, Holt RT, Whitecloud TS, Pienkowski D. Complications of multilevel cervical corpectomies and reconstruction with titanium cages and anterior plating. J Spinal Disord Tech. 2003; 16(1):1-8.

18. Yan D, Wang Z, Deng S, Li J, Soo C. Anterior corpectomy and reconstruction with titanium mesh cage and dynamic cervical plate for cervical spondylotic myelopathy in elderly osteoporosis patients. Arch Orthop Trauma Surg. 2011;131(10):1369-1374. 
19. Wang M, Meng X, Li Y, Feng Y, Chang Z, Hai Y. Effects of antiosteoporosis treatment in the elderly with anterior cervical discectomy and fusion. Acta Orthop Traumatol Turc. 2016;50(2):186-190.

20. Matz PG, Ryken TC, Groff MW. Joint Section on Disorders of the S, Peripheral Nerves of the American Association of Neurological S, and Congress of Neurological S. Techniques for anterior cervical decompression for radiculopathy. J Neurosurg Spine. 2009;11:183-197.

21. Liu Y, Wang H, Li X, et al. Comparison of a zero-profile anchored spacer (ROI-C) and the polyetheretherketone (PEEK) cages with an anterior plate in anterior cervical discectomy and fusion for multilevel cervical spondylotic myelopathy. Eur Spine J. 2016;25(6):1881-1890.

22. Scholz M, Reyes PM, Schleicher P, et al. A new stand-alone cervical anterior interbody fusion device: biomechanical comparison with established anterior cervical fixation devices. Spine. 2009;34(2):156-160.
23. Chin DK, Park JY, Yoon YS, et al. Prevalence of osteoporosis in patients requiring spine surgery: incidence and significance of osteoporosis in spine disease. Osteoporos Int. 2007;18(9):1219-1224.

24. Wang Z, Jiang W, Zhang Z, et al. Comparison of ROI-C and traditional cage with anterior plating for anterior cervical discectomy and fusion. Zhonghua Wai Ke Za Zhi. 2014;52(6):425-430.

25. Melton LJ, Thamer M, Ray NF, et al. Fractures attributable to osteoporosis: report from the National Osteoporosis Foundation. J Bone Miner Res. 1997;12(1):16-23.
Clinical Interventions in Aging

\section{Publish your work in this journal}

Clinical Interventions in Aging is an international, peer-reviewed journal focusing on evidence-based reports on the value or lack thereof of treatments intended to prevent or delay the onset of maladaptive correlates of aging in human beings. This journal is indexed on PubMed Central, MedLine,

\section{Dovepress}

CAS, Scopus and the Elsevier Bibliographic databases. The manuscript management system is completely online and includes a very quick and fair peer-review system, which is all easy to use. Visit http://www.dovepress. $\mathrm{com} /$ testimonials.php to read real quotes from published authors. 